Research Article

\title{
Remote Limb Ischemic Postconditioning Protects against Ischemic Stroke via Modulating Microglia/Macrophage Polarization in Mice
}

\author{
Dong Han, Jue Wang, Lulu Wen, Miao Sun, Hang Liu, and Yan Gao \\ Department of Neurology, Shengjing Hospital of China Medical University, 36 Sanhao Street, Heping District, \\ Shenyang 110004, China \\ Correspondence should be addressed to Yan Gao; cmugaoyan@163.com
}

Received 14 December 2020; Revised 6 February 2021; Accepted 10 February 2021; Published 19 February 2021

Academic Editor: Xiaofeng Yang

Copyright (c) 2021 Dong Han et al. This is an open access article distributed under the Creative Commons Attribution License, which permits unrestricted use, distribution, and reproduction in any medium, provided the original work is properly cited.

\begin{abstract}
Aim. The protection against ischemia/reperfusion injury mediated by remote limb ischemic postconditioning (RIPC) shows great clinical value in ischemic stroke therapy, but the particular mechanism of RIPC remains unclear. Methods. We carried out middle cerebral artery occlusion/reperfusion (MCAO/R) surgery on C57BL/6 male mice. RIPC was generated by 10-minute occlusion followed by the same period of reperfusion of the bilateral hind limb femoral artery and repeated for 3 cycles. Infarct size and neurological score were performed to assess stroke outcomes. Ly6 $\mathrm{C}^{\text {hi }}$ monocytes were quantified in the blood and brain by flow cytometry. Real-time PCR, ELISA, and immunofluorescence were utilized to detect phenotype of proinflammatory M1 and antiinflammatory M2 microglia/macrophage. Nuclear factor $\kappa \mathrm{B}(\mathrm{NF}-\kappa \mathrm{B})$ and peroxisome proliferator-activated receptor $\gamma(\mathrm{PPAR} \gamma)$ levels were detected using Western blot. Results. At 24 and $72 \mathrm{~h}$ after MCAO, RIPC drastically attenuated infarct size and ameliorated the neurological deficits of mice and facilitated transmigration of Ly6C $\mathrm{C}^{\text {hi }}$ monocytes to the brain postischemia reperfusion. Furthermore, RIPC contributed to increased M2 and reduced M1 microglia/macrophage through inhibiting NF- $\kappa \mathrm{B}$ and promoting PPAR $\gamma$ activation. Conclusion. Our results reveal pharmacological effect of RIPC in promoting microglia/macrophage transferring from M1 to M2 phenotype after MCAO/R in mice, which provides theoretical support for the therapeutic effect of RIPC in ischemic stroke.
\end{abstract}

\section{Introduction}

Despite significant advances in medical and surgical intervention, ischemic stroke is still a prominent source of death and long-term disability globally. For patients with acute ischemic stroke, besides timely and effective reperfusion, adjunct therapies are also required to ameliorate the clinical outcomes and decrease infarct sizes. Remote limb ischemic postconditioning (RIPC) is an effective protection strategy that performs ischemic condition on a remote organ (limbs) after cerebral ischemia to protect the brain from ischemia/reperfusion injury in models of experimental stroke. Despite the fact that neuroprotection of RIPC has been validated across several studies $[1,2]$, the underlying mechanisms of its effects have not been clarified.

More and more evidences indicate that microglia/macrophages play a critical function in modulating immune and inflammatory responses [3]. After cerebral ischemia, a large number of microglia are rapidly activated and polarized into two different phenotypes, namely, "classical activation" (M1 phenotype) and "alternative activation" (M2 phenotype) [4]. M1 microglia/macrophages secrete proinflammatory cytokines and neurotoxic mediators, such as tumor necrosis factor- (TNF-) $\alpha$, interleukin- (IL-) $1 \beta$, and IL- 6 . The significant production of these inflammatory cytokines participates in the pathogenesis of stroke and aggravate neuronal 
injury [5]. In M1 microglia/macrophage, nuclear factor $\kappa \mathrm{B}$ $(\mathrm{NF}-\kappa \mathrm{B})$ activation may facilitate the expression of proinflammatory cytokines, leading to neurotoxic outcomes [6]. In comparison to the M1 phenotype, M2 microglia/macrophages exert anti-inflammatory effects, encourage wound healing, and ameliorate tissue repair [5]. Multiple antiinflammatory cytokines, including IL-4, IL-10, transforming growth factor- $\beta$ (TGF- $\beta$ ), and arginase-1 (Arg-1), have been shown to improve inflammatory responses and increase gene expression that plays a role in tissue recovery [7-9]. Thus, swapping microglia/macrophage from an M1 to an M2 phenotype may represent an efficacious treatment strategy for ischemic stroke therapy.

The peroxisome proliferator-activated receptors (PPARs) belong to ligand-induced nuclear receptors. According to the structure, PPARs are divided into three subtypes, including $\operatorname{PPAR} \alpha, \operatorname{PPAR} \beta / \delta$, and $\operatorname{PPAR} \gamma \cdot \operatorname{PPARs}$ bind to ligands, then form heterodimer complex with retinol $\mathrm{X}$ receptors. After that, the complex binds to the PPAR response element, which is upstream of the target gene promoter, and finally modulates gene transcription [10]. PPAR $\gamma$ was first reported to have a significant function in fat formation and glycolipid metabolism [11]. Recently, several researches have shown that activating PPAR $\gamma$ signaling leads to a reduction in inflammation which is related to regulation of the M1/M2 phenotype in Alzheimer's disease and experimental stroke $[9,12]$.

In this study, we explored the therapeutic efficacy of RIPC by identifying the inflammatory cytokines and M1/M2 polarization markers that are present in the cortex of middle cerebral artery occlusion/reperfusion (MCAO/R) mice and also investigated whether the neuroprotective effect is associated with the NF- $\kappa \mathrm{B}$ and PPAR $\gamma$ pathway.

\section{Materials and Methods}

2.1. Animals and Treatment. A total of one hundred and thirty heathy male C57BL/6 mice (20-25 g) were purchased from the Beijing HFK Bioscience Corporation, China. Mice were maintained 5 per cage in a temperature-controlled room $\left(22 \pm 2^{\circ} \mathrm{C}\right)$, at a $12 \mathrm{~h}$ light-dark cycle and a relative humidity of $60 \pm 10 \%$. The mice were randomly allocated to 3 groups: sham, MCAO, and MCAO+RIPC group. Then, all mice were euthanized at $24 \mathrm{~h}$ or $72 \mathrm{~h}$ post-MCAO, respectively. In order to assess neurological deficit, infarct size, and ELISA analyses, $n=6$ for each group were utilized. For realtime PCR, $n=4$ per group. For immunofluorescence, flow cytometry analysis, and Western blot, $n=3$ for each group. Twenty-two mice died from intracranial hemorrhage, and the data from these mice were excluded in the analysis. The protocols for all animal experiments were approved by the Institutional Animal Care and Use Committee of China Medical University (Shenyang, China).

2.2. Focal Cerebral Ischemia. Ischemic stroke was performed as previously described utilizing the mouse model of MCAO with some modifications [13]. Briefly, the right common carotid artery and external and internal carotid artery were exposed, and a silicon-coated monofilament nylon suture (diameter of $0.22 \pm 0.01 \mathrm{~mm}$, Beijing Cinontech Co. Ltd., China) was inserted into the internal carotid artery to occlude the origin of MCA, under isoflurane anesthesia. The filament was removed after $1 \mathrm{~h}$ to initiate reperfusion. During the surgery, core body temperatures were continuously maintained at $36.8-37.2^{\circ} \mathrm{C}$. The sham group was performed the same surgery with the exception of the MCA occlusion.

2.3. Remote Limb Ischemic Postconditioning. RIPC was performed as previously described [14]. In brief, RIPC was accomplished using three cycles of bilateral hind limb ische$\mathrm{mia}(10 \mathrm{~min} /$ cycle, $50 \mathrm{~min}$ total). For every cycle, the proximal region of the hind limbs was secured using a tourniquet for $10 \mathrm{~min}$, followed by reperfusing for $10 \mathrm{~min}$ with the tourniquet released. RIPC was conducted immediately after MCAO/R surgery. The sham and MCAO groups contain all surgical techniques, excluding the cycles of bilateral hind limb ischemia and reperfusion.

2.4. Neurological Scoring. The mice were given scores according to their grades of neurological deficit at $24 \mathrm{~h}$ or $72 \mathrm{~h}$ postreperfusion. The neurological scores were evaluated using the method of Bederson et al. [15]. While being held suspended by the tail, mice that lengthened both forelimbs towards the floor without additional neurological deficits were rated 0 . Mice that fail to extend consistent forelimb fully with the injured hemisphere were assigned grade 1 . When being placed on a smooth surface, mice that had decreased resistance to lateral push on the shoulder towards the paretic side were assigned grade 2 . Mice that circled to the contralateral side were rated 3. Mice that flaccid paralysis without spontaneous movements were allocated a grade of 4 .

2.5. Measurement of Infarct Size. Mice were euthanized and their entire brains were rapidly extracted at $24 \mathrm{~h}$ or $72 \mathrm{~h}$ after surgery. Each brain was dissected and coronally sectioned 5 slices at $2 \mathrm{~mm}$ intervals. Place brain slices in 1\% 2, 3, 5triphenyltetrazolium chloride (TTC, Sangon Biotech Co., Ltd., China) solution and incubate for $10 \mathrm{~min}$ at $37^{\circ} \mathrm{C}$ in the dark [16]. The infarct size on both sides of each section was evaluated utilizing the ImageJ software (version, 1.50; National Institutes of Health, USA). The infarct size was represented as an average percentage from the 5 slices according to this equation:

$$
\begin{aligned}
\text { Infarct size }(\%)= & (\text { contralateral area }- \text { ipsilateral noninfarct } \\
& \text { area }) / \text { contralateral area } \times 100 \% .
\end{aligned}
$$

2.6. ELISA. Tissue samples for ELISA assay were gathered through the ischemic hemisphere after execution. Samples were gathered after centrifuging the tissue homogenate for $10 \mathrm{~min}$ at $5000 \times \mathrm{g}$. The levels of inducible nitric oxide synthase (iNOS), CD86, Arg-1, and CD206 were measured by using corresponding ELISA kits (Shanghai Enzyme-linked Biotechnology Co., Ltd., China). 


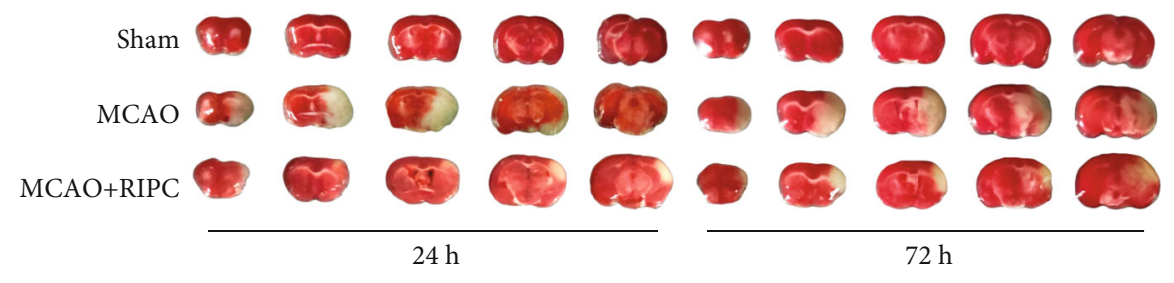

(a)

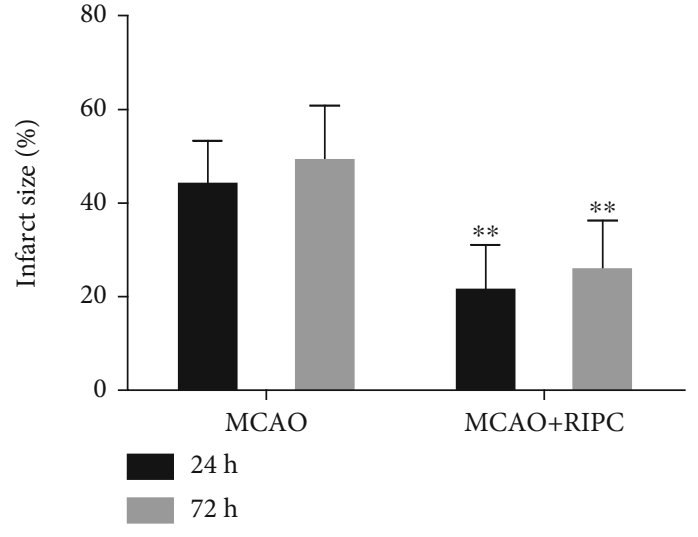

(b)

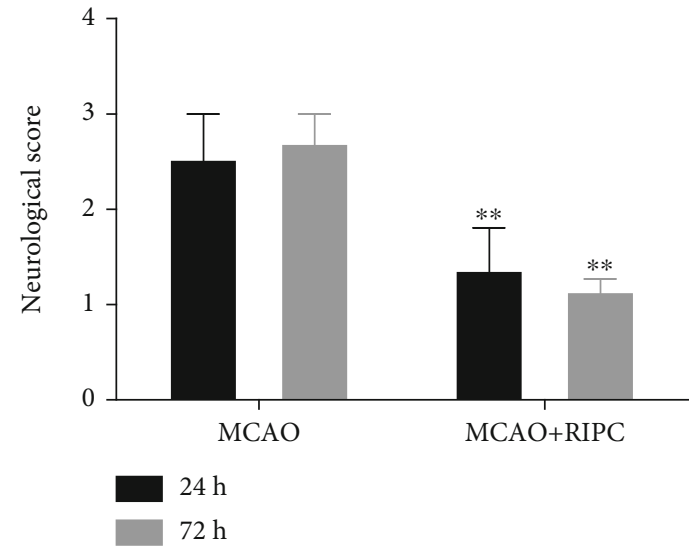

(c)

FIGURE 1: RIPC reduced infarct size and ameliorated neurological behavior at $24 \mathrm{~h}$ and $72 \mathrm{~h}$ after MCAO: (a) representative images of TTCstained brain sections; (b) brain infarct size; (c) neurological function scores. Data presented as mean \pm SD $(n=6) .{ }^{*} P<0.05$ and ${ }^{* *} P<0.01$ vs. the MCAO group.

2.7. Real-Time PCR. The levels of iNOS, TNF- $\alpha$, Arg-1, and TGF- $\beta$ mRNA in each group were determined by real-time PCR as previously described [17]. Total RNA in ischemic brain tissue was removed utilizing Trizol reagent (Vazyme Biotech Co., Ltd., China). As per manufacturer's guidelines, RNA was reverse-transcribed into cDNA utilizing HiScript II Q RT SuperMix kit; real-time PCR was conducted through ChamQ SYBR qPCR Master Mix kit in quantitative PCR Mastercycler (Eppendorf, Germany). The two kits mentioned above were purchased from Vazyme Biotech Co., Ltd. (China). The primer sequences (Sangon Biotech Co., Ltd., China) were as follows:

iNOS: F: AATGGCAACATCAGGTGGCCATCACT, R: GCTGTGTGTCACAGAAGTCTCGAACTC;

TNF- $\alpha$ : F: GCACCACCATCAAGGACTCA, R: TCGA GGCTCCAGTGAATTCG;

Arg-1: F: GAACACGGCAGTGGCTTTAAC, R: TGCT TAGTTCTGTCTGCTTTGC;

TGF- $\beta$ : F: TGGCTGAACCAAGGAGACG, R: GCAGTG AGCGCTGAATCGA;

$\beta$-Actin: F: CATCCGTAAAGACCTTTGCCAAC, R: ATGGTGCCACCGATCCACA.

2.8. Immunofluorescence. At $24 \mathrm{~h}$ or $72 \mathrm{~h}$ after surgery, animals were deeply anaesthetized and perfused with saline, followed by $4 \%$ paraformaldehyde. The brains were gathered and dehydrated in 30\% sucrose solution overnight. After that, the brains were placed in OCT and cut into $20 \mu \mathrm{m}$ frozen segments. The slices were placed in blocking buffer in $8 \%$ goat serum for $2 \mathrm{~h}$ at room temperature and then treated with a primary antibody: mouse anti-Iba1 (1:100, Santa
Cruz Biotechnology, USA), rabbit anti-CD16 (1:100, Abcam, USA), and rabbit anti-CD206 (1:100, Abcam, USA) at $4^{\circ} \mathrm{C}$ overnight. Membranes were washed with PBST three times $(0.1 \%$ tween 20 in PBS); the tissue sections were treated with FITC- or Cy3-conjugated secondary antibody $(1: 100$, BIOSS, China) and at room temperature for $2 \mathrm{~h}$. Finally, nuclei were costained with DAPI (Beyotime Institute of Biotechnology, China) for $30 \mathrm{~min}$. Images were obtained through a laser scanning confocal microscope (LSM700, Carl Zeiss, Germany) and assessed using ImageJ software. The stained cells were counted in the ipsilateral cortex penumbra under 400x magnification.

2.9. Flow Cytometry Analysis. The brain was removed, and the ischemic hemisphere was collected for flow cytometry. Monocyte classification by phenotypic analysis through expression of cell surface antigens was conducted using flow cytometry [18]. All antibodies were bought through BioLegend (USA), including fluorescein isothiocyanate (FITC) anti-mice Ly6C (128005), phycoerythrin (PE) anti-mice CD11b (101207), and PE-Cy5 conjugate (PE/Cy5) antimice CD45 (103109). Cells were analyzed on a Miltenyi Biotec MACSQuant flow cytometer (Germany). Cells were gated on the scatter plots of forward scatter (FSC-H) and side scatter (SSC-H), with the exclusion of debris and cell aggregates. The total number of leukocytes was gated as CD $45^{\mathrm{hi}}$ populations. Monocytes were identified as CD $45^{\text {hi }} \mathrm{CD}_{11 b^{+}} \mathrm{Ly}_{6 \mathrm{C}^{+}}$ populations and further separated as Ly6C $C^{\text {hi }}$ or Ly6 $C^{\text {lo }}$.

2.10. Western Blot. To identify NF- $\kappa \mathrm{B}$ and $\operatorname{PPAR} \gamma$ protein levels in ischemic brain, protein from the nucleus and 

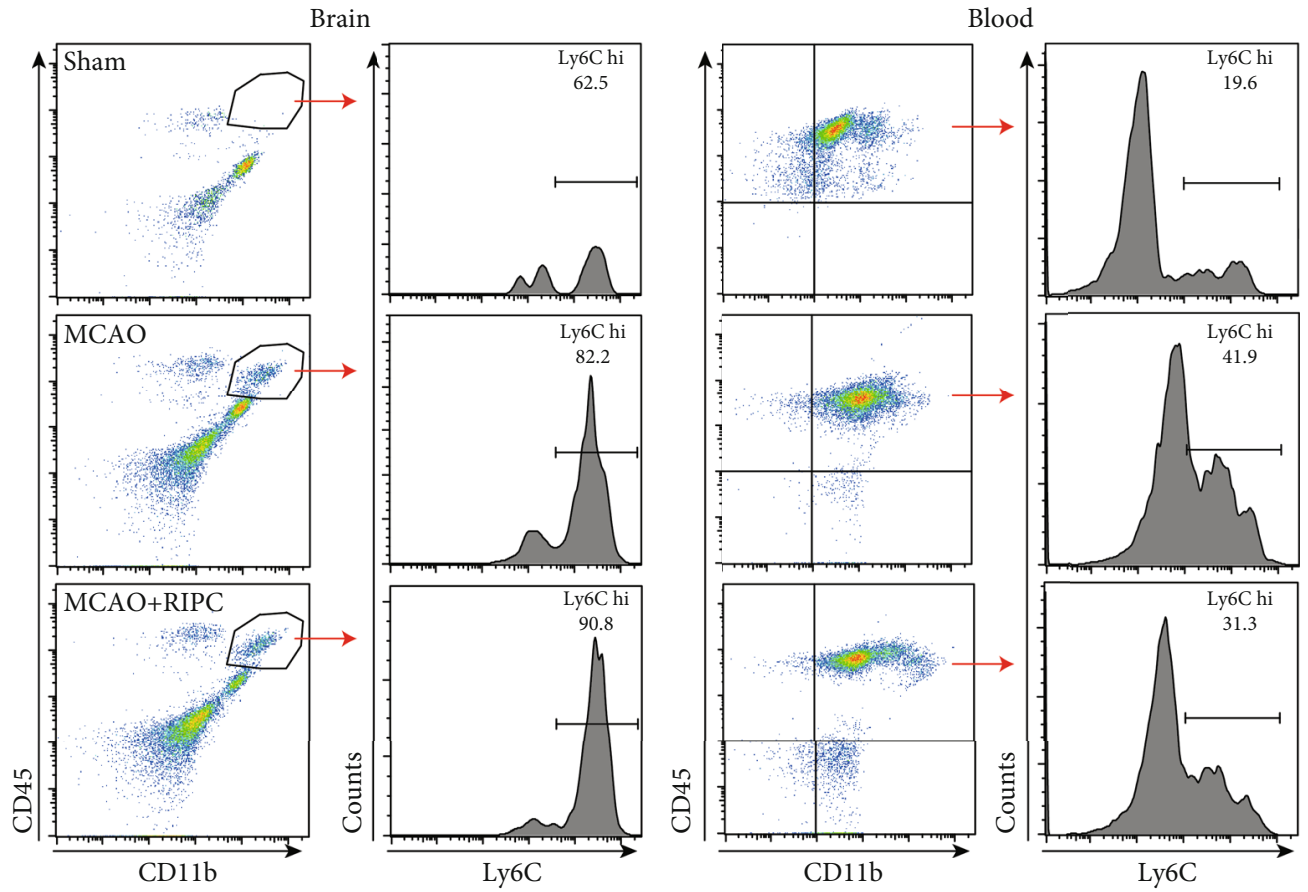

(a)

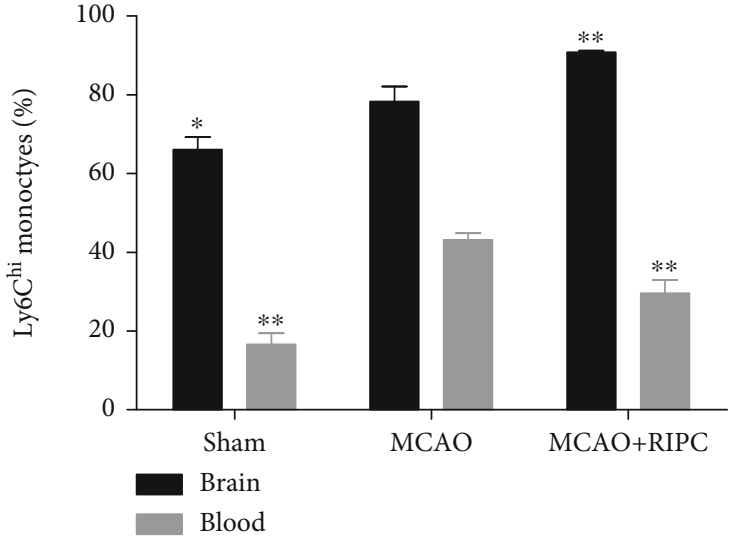

(b)

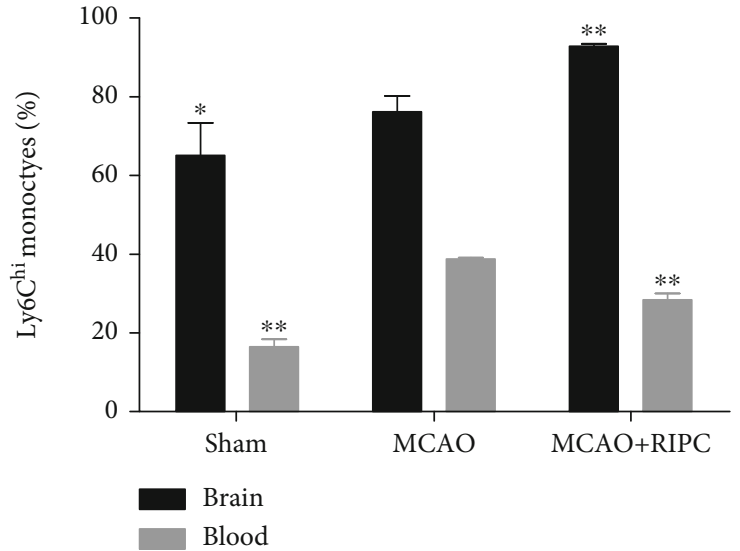

(c)

FIGURE 2: RIPC accelerated the infiltration of Ly6 $\mathrm{C}^{\text {hi }}$ monocytes into ischemic brain at $24 \mathrm{~h}$ and $72 \mathrm{~h}$ after MCAO. (a) Representative gating strategy for flow cytometric analysis of Ly6C ${ }^{\text {hi }}$ monocytes in the brain and blood at $24 \mathrm{~h}$. Quantification of the percentage of Ly6C hi monocytes in the brain and blood at $24 \mathrm{~h}(\mathrm{~b})$ and $72 \mathrm{~h}(\mathrm{c})$. Data presented as mean $\pm \mathrm{SD}(n=3) .{ }^{*} P<0.05$ and ${ }^{* *} P<0.01$ vs. the MCAO group.

cytoplasm was isolated utilizing the Nuclear and Cytoplasmic Extract Kit (Solarbio, China). Next, concentration of total protein was evaluated using BCA assay (Applygen Technologies Inc., China). Equivalent levels of protein were separated through SDS-PAGE and transferred onto polyvinylidene fluoride (PVDF) membranes. After treatment with blocking buffer ( $5 \%$ skim milk in TBST) for $1.5 \mathrm{~h}$ at room temperature, the membranes were probed with primary antibodies targeting PPAR $\gamma(1: 1000$, Cell Signaling Technology, USA), NF$\kappa \mathrm{B}(1: 1000$, Proteintech Group, USA), $\beta$-actin $(1: 3000$, Abways Technology, China), and H3 (1: 1000, Abways Technology, China) overnight at $4^{\circ} \mathrm{C}$. After being washed three times with TBST, the membranes were probed with horse- radish peroxidase- (HRP-) conjugated secondary antibodies (1:5000, Abways Technology, China) for $2 \mathrm{~h}$. Relative band densities were subsequently observed using ECL kit (Applygen Technologies Inc., China) on a chemiluminescence imaging system (Bio-Rad, USA). Protein levels were measured with ImageJ, and the data was normalized to anti- $\beta$ actin or anti-H3.

2.11. Statistical Analysis. All data were evaluated or quantified in a completely blind manner. IBM SPSS Statistics v19.0 software was used to perform statistical analyses. Statistical variation between groups was determined utilizing oneway analysis of variance (ANOVA). The neurological scores 


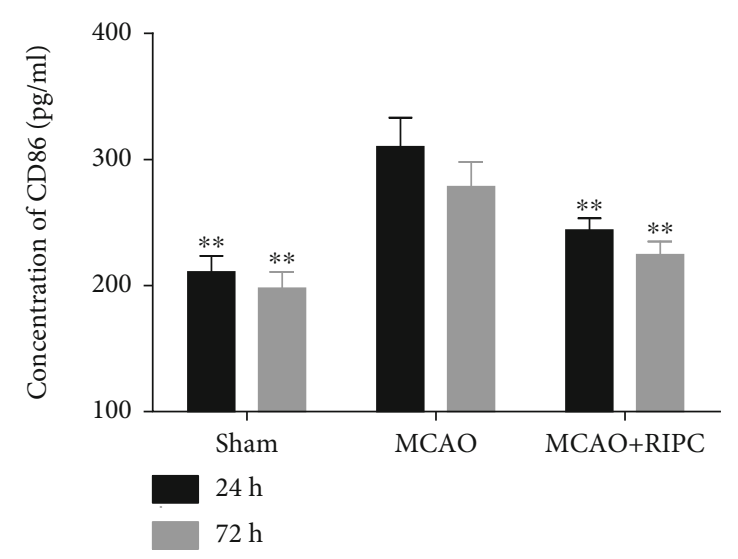

(a)

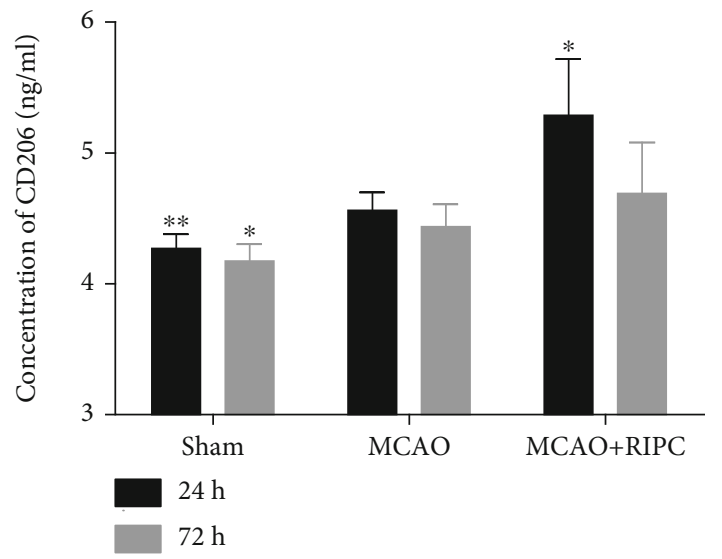

(c)

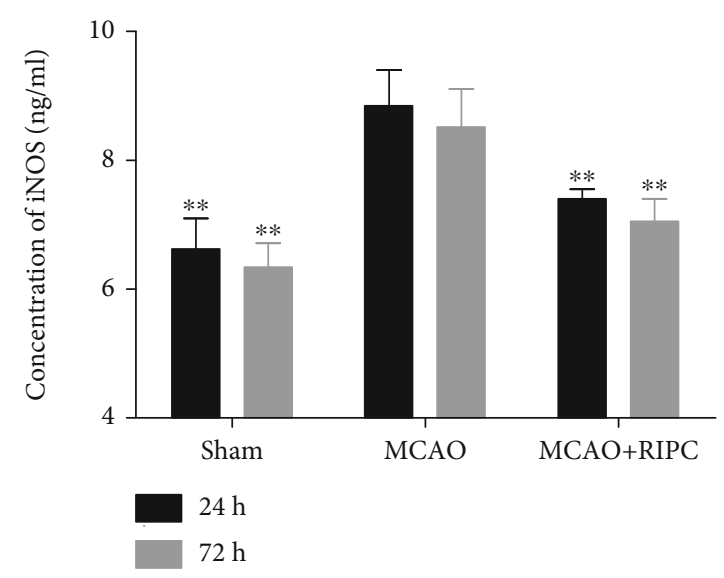

(b)

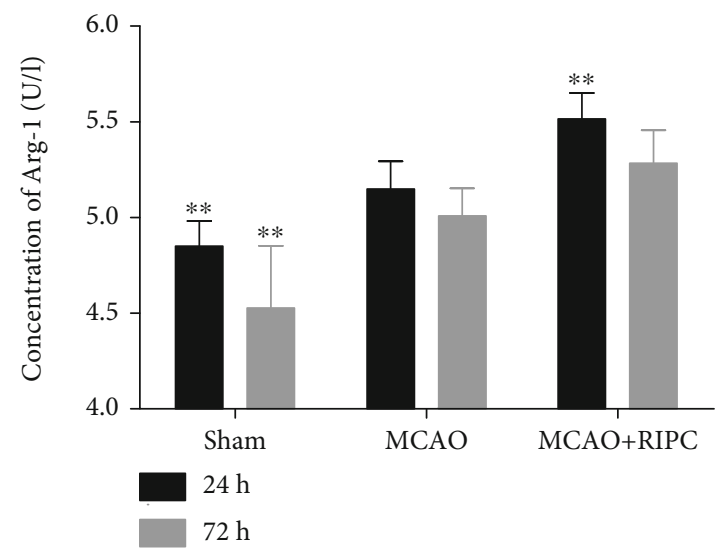

(d)

FIgURE 3: RIPC downregulated the expressions of CD86 and iNOS, while it upregulated the expressions of CD206 and Arg-1 in ischemic brain at $24 \mathrm{~h}$ or $72 \mathrm{~h}$ after MCAO. The level of cerebral homogenate CD86 (a), iNOS (b), CD206 (c), and Arg-1 (d) at $24 \mathrm{~h}$ or $72 \mathrm{~h}$ after $\mathrm{MCAO}(n=6)$. Values were shown as mean $\pm \mathrm{SD}^{*} P<0.05$ and ${ }^{* *} P<0.01$ vs. the MCAO group.

and real-time PCR were compared with other groups using the Mann-Whitney $U$ test. The comparative variation was significant at $P<0.05$. Statistical figures were presented using ImageJ, Photoshop CS6, and GraphPad Prism version 5.0. The data was presented as mean $\pm \mathrm{SD}$.

\section{Result}

3.1. RIPC Attenuated Ischemic Outcome Both at $24 \mathrm{H}$ and $72 \mathrm{H}$ in Mice after MCAO. The infarct size and neurological defect were detected in different groups at $24 \mathrm{~h}$ and $72 \mathrm{~h}$ postreperfusion. As depicted in Figure 1(b), the infarct size of the $\mathrm{MCAO+RIPC}$ group significantly decreased in comparison to the MCAO group at $24 \mathrm{~h}$ and $72 \mathrm{~h}(P<0.01)$. The consequence of RIPC on neurological dysfunction stimulated by $\mathrm{MCAO}$ was assessed. The scores of the sham group were zero, which showed no neurological deficits. The scores increased in the MCAO group, while those of the MCAO + RIPC group decreased significantly in comparison with the MCAO group at $24 \mathrm{~h}$ and $72 \mathrm{~h}(P<0.01$, Figure $1(\mathrm{c}))$. These results suggested that RIPC reduced infarct size and improved neurological deficit score at $24 \mathrm{~h}$ and $72 \mathrm{~h}$ after stroke in mice.
3.2. RIPC Upregulated Ly6C ${ }^{\text {hi }}$ Monocyte Infiltration into Ischemic Brain in Mice after MCAO. We use CD45 and $\mathrm{CD} 11 \mathrm{~b}$ to distinguish monocytes $\left(\mathrm{CD} 45^{+} \mathrm{CD} 11 \mathrm{~b}^{+}\right)$and further gated to quantify inflammatory monocytes (Ly6 $\mathrm{C}^{\text {hi }}$ ) which have a protective role in stroke progression (GarciaBonilla et al. 2018). Based on flow cytometry assessment, increased amount of monocytes occurred in ischemic brain postcerebral ischemia when compared to the sham group (Figure 2(a)). Further analyses indicated that the percentage of Ly6C $\mathrm{C}^{\text {hi }}$ monocytes in the MCAO+RIPC group significantly increased in ischemic brain and decreased in peripheral blood in comparison to the MCAO group at $24 \mathrm{~h}$ and $72 \mathrm{~h}$ after stroke $(P<0.01$, Figures $2(\mathrm{~b})$ and $2(\mathrm{c}))$. These results indicated that RIPC accelerated the infiltration of Ly6 $\mathrm{C}^{\text {hi }}$ monocytes into ischemic brain in mice after MCAO.

3.3. RIPC Regulated CD86, iNOS, CD206, and Arg-1 Expressions in Mice after MCAO. Overexpression of biomarkers such as CD86 and iNOS is associated with proinflammatory phenotype microglia/macrophage (M1), whereas CD206 and Arg-1 were selected as antiinflammatory phenotype microglia/macrophage (M2) marker. As shown in Figure 3 ELISA results, the levels of 


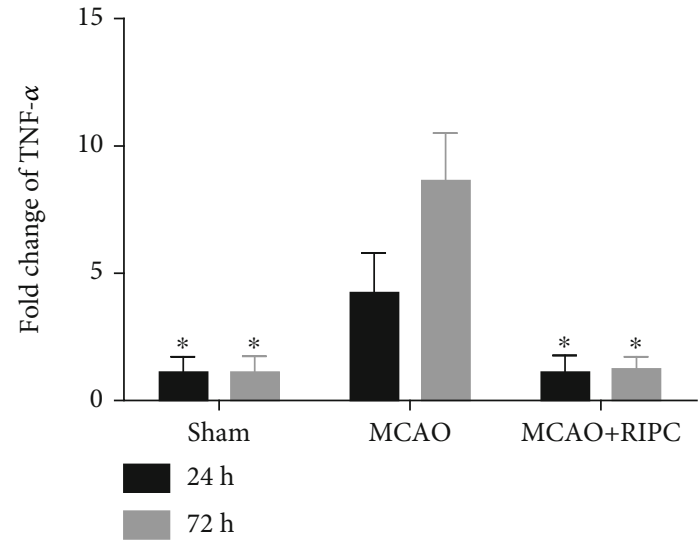

(a)

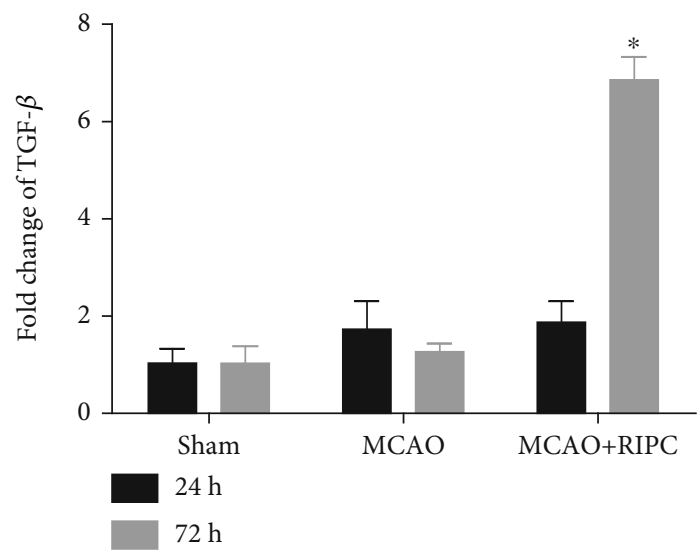

(c)

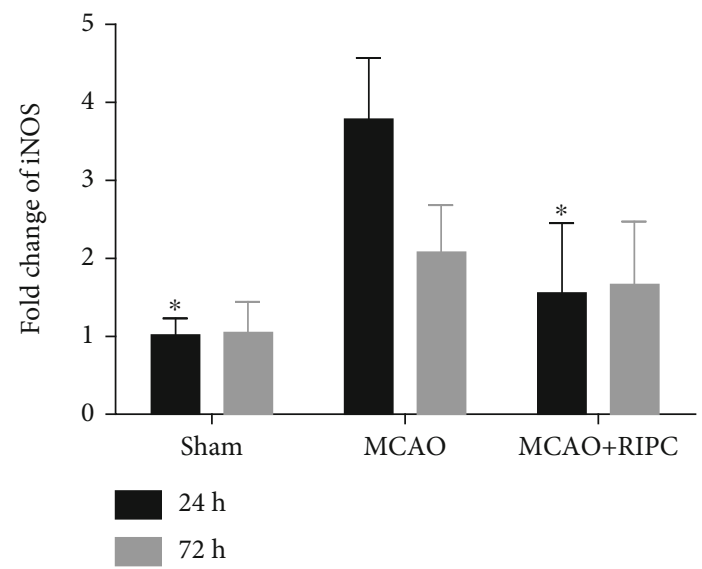

(b)

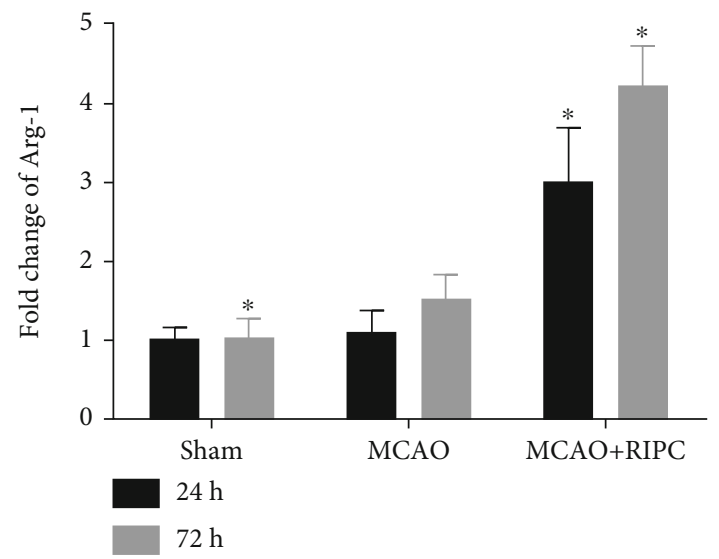

(d)

FIgURE 4: RIPC suppressed the mRNA expressions of TNF- $\alpha$ and iNOS and promoted the mRNA expressions of TGF- $\beta$ and Arg- 1 in ischemic brain at $24 \mathrm{~h}$ and $72 \mathrm{~h}$ after MCAO. Real-time PCR of TNF- $\alpha$ (a), iNOS (b), TGF- $\beta$ (c), and Arg-1 (d) at $24 \mathrm{~h}$ and $72 \mathrm{~h}$ after $\operatorname{MCAO}(n=4)$. Values were shown as mean $\pm \mathrm{SD}^{*} P<0.05,{ }^{* *} P<0.01 v$ s. the MCAO group.

both CD86, iNOS, CD206, and Arg-1 were higher in the $\mathrm{MCAO}$ group at $24 \mathrm{~h}$ and $72 \mathrm{~h}$ in comparison to the sham group $(P<0.01)$. In comparison to the MCAO group, the dramatic enhancement of CD86 and iNOS in brain tissue was inhibited by RIPC $(P<0.01$, Figures $3(a)$ and $3(b))$. After RIPC, the expression of CD206 and Arg-1 in brain tissue raised significantly at $24 \mathrm{~h}$ post-MCAO (Figures $3(\mathrm{c}$ ) and $3(\mathrm{~d})$ ), whereas the trends were not apparent at $72 \mathrm{~h}$ postMCAO. These results showed that RIPC downregulated the expressions of CD86 and iNOS, while it upregulated the expressions of CD206 and Arg-1.

3.4. RIPC Regulated TNF- $\alpha$, iNOS, TGF- $\beta$, and Arg- 1 mRNA Expressions in Mice after MCAO. To explore the outcome of RPIC on microglia/macrophage polarization, we determined the mRNA expression of TNF- $\alpha$, iNOS (both M1-related factors), TGF- $\beta$, and Arg-1 (both M2-related factors) by realtime PCR in ischemic brain. As shown in Figure 3, TNF- $\alpha$, iNOS, and Arg- 1 increased significantly in the MCAO group compared to the sham group at $24 \mathrm{~h}$ or $72 \mathrm{~h}$ post-MCAO $(P<0.05)$. In the MCAO+RIPC group, the mRNA expression of TNF- $\alpha$ showed a significant decrease at $24 \mathrm{~h}$ and $72 \mathrm{~h}$ post-MCAO $(P<0.05$, Figure $4(\mathrm{a}))$, while the mRNA level of iNOS significantly reduced at $24 \mathrm{~h}$ post-MCAO $(P<0.05$, Figure $4(\mathrm{~b}))$. In contrast, TGF- $\beta$ and Arg- 1 were elevated dramatically in the MCAO+RIPC group in comparison to the MCAO group at $72 \mathrm{~h}$ post-MCAO $(P<0.05$, Figures $4(\mathrm{c})$ and $4(\mathrm{~d}))$. Taken together, these results suggested that RIPC suppressed the mRNA expressions of M1related factors (TNF- $\alpha$ and iNOS) and promoted the mRNA expressions of M2-related factors (TGF- $\beta$ and Arg-1).

3.5. RIPC Regulated Microglia/Macrophage Polarization in Mice after MCAO. To further evaluate the polarization of microglia/macrophage in ischemic brain, we used Iba-1 to mark resting and activated microglia/macrophage and CD16 and CD206 to mark M1 and M2, respectively [19, 20]. The immunofluorescence staining result showed that levels of the M1 marker CD16 in the MCAO group were higher in $\mathrm{Ibal}^{+}$cells at $24 \mathrm{~h}$ and $72 \mathrm{~h}$ post-MCAO (Figure 5(b)). When compared to the MCAO group, CD16 and Ibal coexpression was lower in the MCAO+RIPC group $(P<0.01$, Figure 5(b)). In contrast, levels of the M2 marker $\mathrm{CD} 206$ heightened in the MCAO+RIPC group in comparison to the MCAO group at $24 \mathrm{~h}$ and $72 \mathrm{~h}$ post-MCAO (Figure 5(c)). Moreover, the MCAO+RIPC group showed 

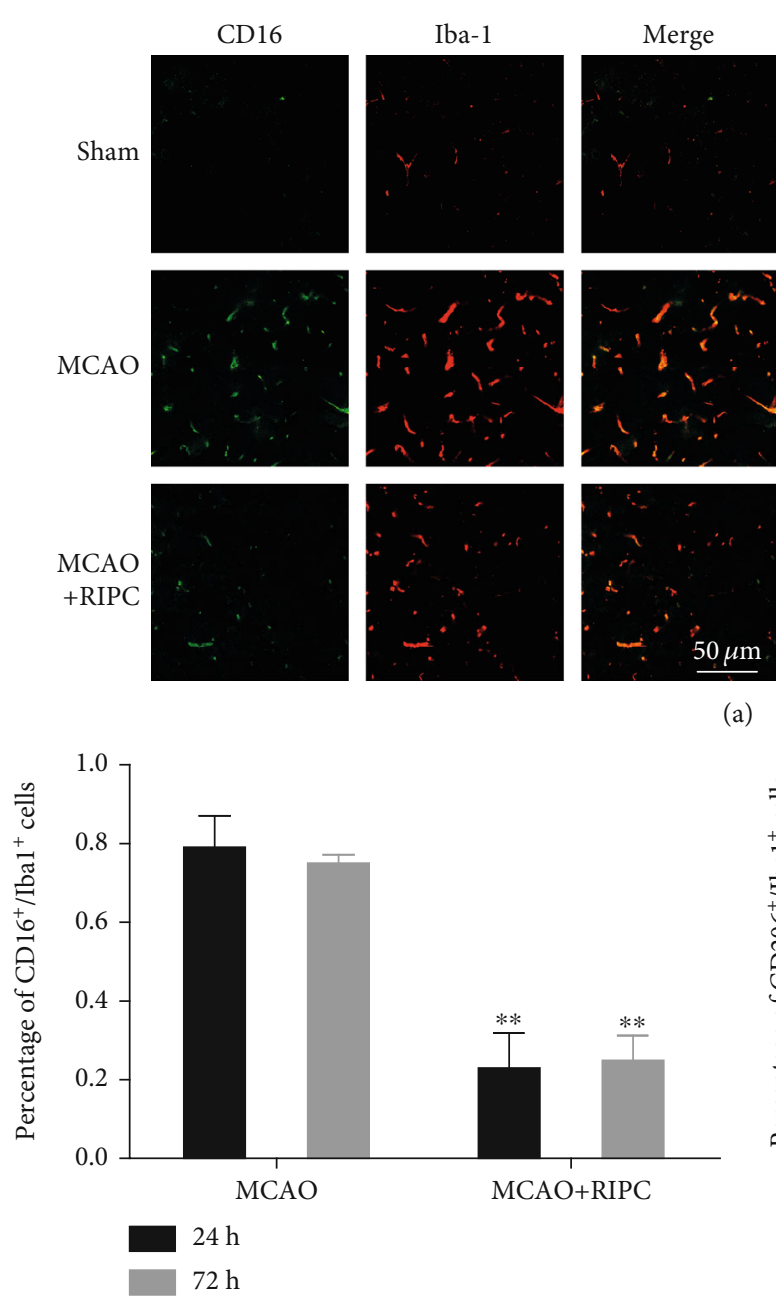

(b)
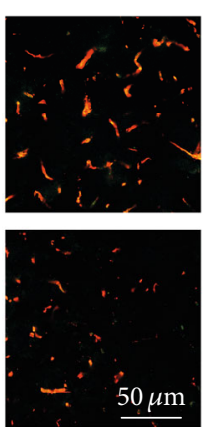

(a)
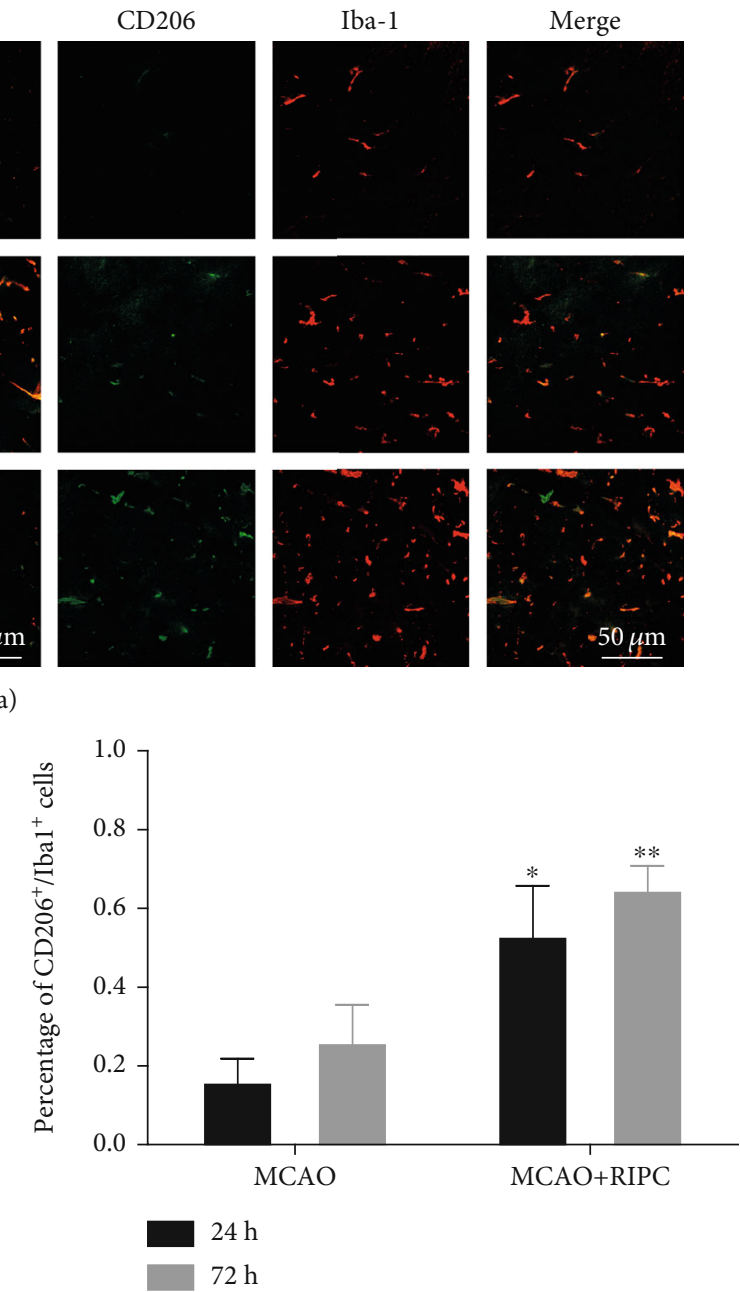

(c)

FIGURE 5: RIPC inhibited proinflammatory microglia and facilitated anti-inflammatory microglia polarization in ischemic brain at $24 \mathrm{~h}$ and $72 \mathrm{~h}$ after MCAO: (a) representative immunofluorescence staining images of brain sections at $24 \mathrm{~h}$ after MCAO; (b) quantification of the percentage of CD16 $6^{+} / \mathrm{Iba}-1^{+}$cells at 24 and $72 \mathrm{~h}$ after MCAO; (c) quantification of the percentage of CD206 $/ \mathrm{Iba}-1^{+}$cells at 24 and $72 \mathrm{~h}$ after MCAO. Data presented as mean $\pm \mathrm{SD}(n=3) .{ }^{*} P<0.05$ and ${ }^{* *} P<0.01 v s$. the MCAO group.

more significant difference than the MCAO group at $72 \mathrm{~h}$ $(P<0.01)$. These data suggested that RIPC inhibited proinflammatory microglia and facilitated anti-inflammatory microglia polarization in MCAO mice.

3.6. RIPC Regulated PPAR $\gamma$ and $N F-\kappa B$ Shifting from the Nuclei to Cytoplasm in Mice after MCAO. In comparison to the sham group, expression of NF- $\kappa \mathrm{B}$ substantially decreased in the cytoplasm but heightened in the nucleus of the MCAO group. After RIPC treatment, cytoplasmic NF- $\kappa \mathrm{B}$ increased and nuclear NF- $\kappa \mathrm{B}$ reduced (Figures $6(\mathrm{a})$ and $6(\mathrm{~b})$ ). Consistently, the MCAO+RIPC group showed more significant difference than the MCAO group at $72 \mathrm{~h}(P<0.01)$.

In the MCAO group, PPAR $\gamma$ levels drastically decreased in the cytoplasm $(P<0.01)$ but enhanced in nucleus in comparison to the sham group. After RIPC therapy, PPAR $\gamma$ reduced in the cytoplasm while increased in the nucleus (Figures 6(c) and 6(d)). The difference between the MCAO + RIPC group and MCAO group was significant at $72 \mathrm{~h}$
$(P<0.01)$. Therefore, RIPC inhibited nuclear NF- $\kappa \mathrm{B}$ and cytoplasmic PPAR $\gamma$ levels, while it upregulated cytoplasmic $\mathrm{NF}-\kappa \mathrm{B}$ and nuclear PPAR $\gamma$ expression after MCAO. In other words, RIPC repressed NF- $\kappa$ B shifting from the cytoplasm to the nucleus and stimulated PPAR $\gamma$ shifting from the nucleus to the cytoplasm.

\section{Discussion}

In this present study, our findings indicated that RIPC substantially decreased infarct size, attenuated neurological impairment, promoted $\mathrm{Ly}_{6 \mathrm{C}} \mathrm{Ci}^{\mathrm{m}}$ monocyte infiltration into the brain, and suppressed NF- $\kappa \mathrm{B}$-mediated inflammatory response. Furthermore, this neuroprotection was related to promotion of microglia/macrophage M2 polarization through upregulating PPAR $\gamma$ nuclear translocation.

Ren et al. were the first to describe that RIPC conducted on the ipsilateral hind limb reduced the cerebral infarct size after focal cerebral ischemia [21]. An increased number of 


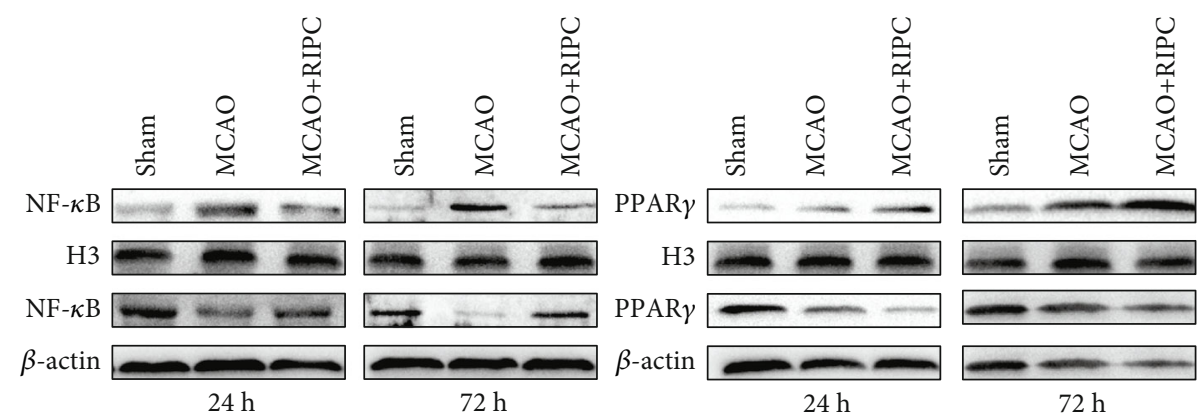

(a)

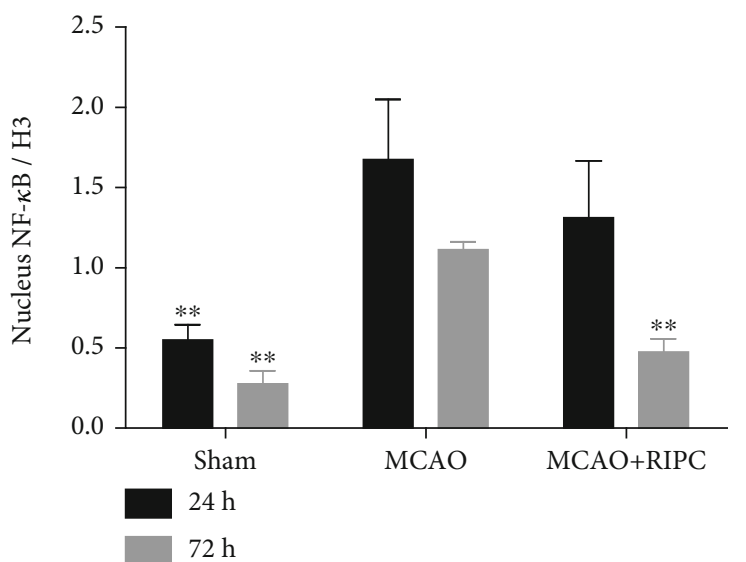

(b)

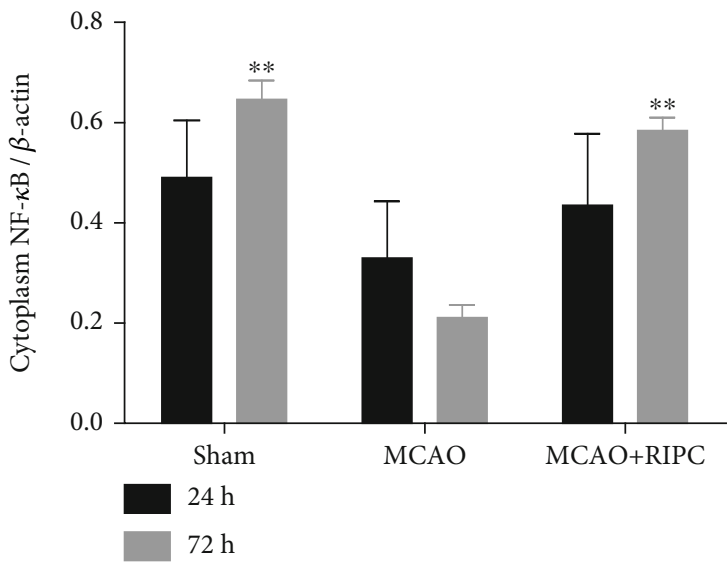

(d)

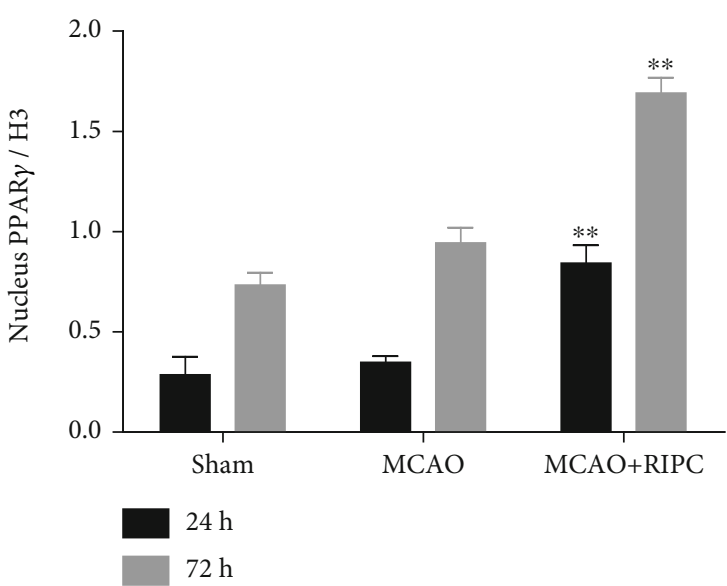

(c)

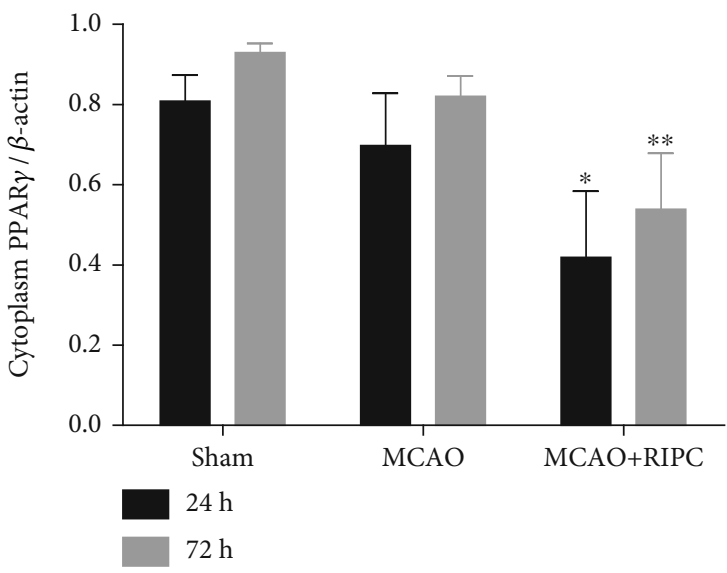

(e)

FIGURE 6: RIPC repressed NF- $\kappa$ B shifting from the cytoplasm to nucleus and stimulated PPAR $\gamma$ shifting from the nucleus to cytoplasm in ischemic brain at $24 \mathrm{~h}$ or $72 \mathrm{~h}$ after MCAO. Western blot of the nucleus NF- $\kappa \mathrm{B}$ (a), cytoplasm NF- $\kappa \mathrm{B}$ (b), nucleus PPAR- $\gamma$ (c), and cytoplasm PPAR $-\gamma(\mathrm{d})$ in ischemic brain. Data were shown as mean $\pm \mathrm{SD}(n=3) .{ }^{*} P<0.05$ and ${ }^{* *} P<0.01$ vs. the MCAO group.

reports indicate that PIPC is protective from stroke-induced brain injury $[22,23]$. Consistent with prior studies, our findings indicated that RIPC not only decreased cerebral infarct size but also promoted neurological role in early stage postischemic stroke.

Monocytes, which are recruited from peripheral blood after ischemic brain injury, can be separated into two categories in mice: inflammatory and patrolling monocytes [24]. The two common types of murine monocytes are classified by their level of Ly6C expression: Ly6C hi and Ly6C ${ }^{\text {lo }}$. Chu at el suggested that $\mathrm{Ly}_{6 \mathrm{C}}{ }^{\mathrm{hi}}$ monocytes exerted an acute pro- tective influence after the establishment of ischemic stroke and functional deficit that involved promotion of M2 macrophage polarization [16]. Using flow cytometry, we characterized the profile of monocytes circulating in the blood and entering the brain early after stroke. There was a profound increase in the proportion of brain monocytes after stroke. Our data also showed that Ly6 $\mathrm{C}^{\text {hi }}$ monocytes entered the circulation and then the ischemic brain during the acute poststroke period. RIPC improved the percentage of Ly $6 \mathrm{C}^{\text {hi }}$ monocytes in ischemic brain, resulting in beneficial outcome after cerebral ischemia. 
Recently, studies have demonstrated that microglia/macrophage can function as double-edged swords in many diseases, depending on their phenotypes [25]. For example, the M2 phenotype ameliorated neuronal survival and tissue repair, while the M1 phenotype hastens neuronal necrosis and aggravates inflammation [26, 27]. And prior analyses have suggested that regulating the polarization of microglia, inhibiting M1 phenotype, and promoting M2 phenotype lead to neuroprotective effects in animal models of ischemic stroke $[28,29]$. Similarity, our immunofluorescence data indicated that RIPC increased microglia/macrophage polarization towards M2 phenotype rather than M1 phenotype, as shown by a reduction of $\mathrm{CD} 16^{+} / \mathrm{Iba}-1^{+}$cells and increased CD206 $/ \mathrm{Iba}-1^{+}$cells at $24 \mathrm{~h}$ and $72 \mathrm{~h}$ postreperfusion. Consistent with immunofluorescence results, RIPC promoted the M2-related factor (TGF- $\beta$ and Arg-1) mRNA expression and inhibited M1-related factor (TNF- $\alpha$ and iNOS) mRNA expression. ELISA results indicated that RIPC decreased levels of M1 markers (CD86 and iNOS) and increased M2 marker expression (CD206 and Arg-1) at $24 \mathrm{~h}$ and $72 \mathrm{~h}$ post-MCAO/R.

Overall, these results together suggested that RIPC was protective towards ischemic stroke by encouraging microglia/macrophage polarization to M2 phenotype in MCAO/R mice.

In addition, we investigated the mechanisms of RIPC regulating inflammatory response and microglia/macrophage polarization. The NF- $\kappa \mathrm{B}$ signaling pathway has a vital function in stimulating proinflammatory gene expression [30, 31]. Normally, NF- $\kappa$ B is present within the cytoplasm, where it interacts with and binds to inhibitory proteins and stays inactive. In our experiment, cytoplasmic NF- $\kappa \mathrm{B}$ was reduced and the protein levels of NF- $\kappa \mathrm{B}$ in the nucleus were increased, while RIPC reversed the NF- $\kappa$ B signaling pathway activation, which suggested the anti-inflammatory effects of PIPC. PPAR $\gamma$ is constitutively expressed in microglia/macrophage, acting as a key regulator of microglia/macrophage activation [32]. Activating the PPAR $\gamma$ signaling pathway has a protective function as it reduces neuroinflammation in several diseases (i.e., Alzheimer's disease, Parkinson's disease, and stroke) $[12,33,34]$. There is also evidence showing that activating PPAR $\gamma$ increases the expression of M2 genes (i.e., Arg-1, CD206, and IL-10) in microglia/macrophage [35]. In our study, RIPC after MCAO/R increased PPAR $\gamma$ nuclear translocation, which contributed to the improvement of M2 gene transcription. Thus, we concluded that the neuroprotective effect of RIPC is related to regulation of microglia polarization regulated by the PPAR $\gamma$ pathway. There are many other potential mechanisms of RIPC protecting against ischemic stroke. A study showed that trained immunity regulators are upregulated in liver ischemic pre- and postconditioning [36]. After cerebral ischemia, RIPC might upregulate the canonical and noncanonical inflammasomes to exert protective effect. In addition to the roles of endothelial cells (ECs) in physiological processes, ECs actively participate in both innate and adaptive immune responses [37]. The regulation of RIPC on ECs is also an interesting research direction. Thus, the effects of RIPC on the regulation of trained immunity are worthy of our research in future experiments.

\section{Conclusion}

We demonstrated that RIPC inhibited the inflammatory reaction and enhanced the postischemic M2 polarization of microglia/macrophage after MCAO/R. Furthermore, our results suggested that the underlying mechanisms were mediated through the NF- $\kappa \mathrm{B}$ and PPAR $\gamma$ pathway. This finding suggests the pharmacological efficacy of RIPC and provides evidence of clinical application of RIPC in ischemic stroke therapy.

\section{Data Availability}

The data used to support the findings of this study are included within the article.

\section{Conflicts of Interest}

The authors declare that the research was conducted in the absence of any commercial or financial relationships that could be construed as a potential conflict of interest.

\section{Authors' Contributions}

Dong Han and Yan Gao conceived and designed the experiments. Dong Han, Jue Wang, and Lulu Wen conducted the experiments and wrote the manuscript. Dong Han, Miao Sun, and Hang Liu provided and analyzed some of the data. Han Dong and Yan Gao reviewed and revised the manuscript. All authors contributed to the article and approved the submitted version.

\section{Acknowledgments}

This study was sponsored by Key projects of Liaoning Natural Science Foundation (No. 20170541053).

\section{References}

[1] G. Chen, X. Ye, J. Zhang et al., "Limb remote ischemic postconditioning reduces ischemia-reperfusion injury by inhibiting NADPH oxidase activation and MyD88-TRAF6P38MAP-kinase pathway of neutrophils," International Journal of Molecular Sciences, vol. 17, no. 12, 2016.

[2] G. Pignataro, E. Esposito, R. Sirabella et al., "nNOS and p-ERK involvement in the neuroprotection exerted by remote postconditioning in rats subjected to transient middle cerebral artery occlusion," Neurobiology of Disease, vol. 54, pp. 105$114,2013$.

[3] V. H. Perry, J. A. Nicoll, and C. Holmes, "Microglia in neurodegenerative disease," Nature Reviews. Neurology, vol. 6, no. 4, pp. 193-201, 2010.

[4] X. Y. Xiong, L. Liu, and Q. W. Yang, "Functions and mechanisms of microglia/macrophages in neuroinflammation and neurogenesis after stroke," Progress in Neurobiology, vol. 142, pp. 23-44, 2016.

[5] A. R. Patel, R. Ritzel, L. D. McCullough, and F. Liu, "Microglia and ischemic stroke: a double-edged sword," International journal of physiology, pathophysiology and pharmacology, vol. 5, no. 2, pp. 73-90, 2013. 
[6] J. Park, J. S. Min, B. Kim et al., "Mitochondrial ROS govern the LPS-induced pro-inflammatory response in microglia cells by regulating MAPK and NF- $\kappa \mathrm{B}$ pathways," Neuroscience Letters, vol. 584, pp. 191-196, 2015.

[7] X. Hu, R. K. Leak, Y. Shi et al., "Microglial and macrophage polarization-new prospects for brain repair," Nature Reviews. Neurology, vol. 11, no. 1, pp. 56-64, 2015.

[8] Y. Ma, J. Wang, Y. Wang, and G. Y. Yang, "The biphasic function of microglia in ischemic stroke," Progress in Neurobiology, vol. 157, pp. 247-272, 2017.

[9] J. Pan, J. L. Jin, H. M. Ge et al., "Malibatol A regulates microglia M1/M2 polarization in experimental stroke in a PPAR $\gamma$ dependent manner," Journal of Neuroinflammation, vol. 12, no. $1,2015$.

[10] C. Giaginis, G. Tsourouflis, and S. Theocharis, "Peroxisome proliferator-activated receptor-gamma (PPAR-gamma) ligands: novel pharmacological agents in the treatment of ischemia reperfusion injury," Current Molecular Medicine, vol. 8, no. 6, pp. 562-579, 2008.

[11] P. Tontonoz, E. Hu, and B. M. Spiegelman, "Stimulation of adipogenesis in fibroblasts by PPAR 2 , a lipid-activated transcription factor," Cell, vol. 79, no. 7, pp. 1147-1156, 1994.

[12] S. Mandrekar-Colucci, J. C. Karlo, and G. E. Landreth, "Mechanisms underlying the rapid peroxisome proliferator-activated receptor- $\gamma$-mediated amyloid clearance and reversal of cognitive deficits in a murine model of Alzheimer's disease," The Journal of Neuroscience, vol. 32, no. 30, pp. 10117-10128, 2012.

[13] M. S. Spychala, V. R. Venna, M. Jandzinski et al., "Age-related changes in the gut microbiota influence systemic inflammation and stroke outcome," Annals of Neurology, vol. 84, no. 1, pp. 23-36, 2018.

[14] H. Guo, L. Zhao, B. Wang et al., "Remote limb ischemic postconditioning protects against cerebral ischemia- reperfusion injury by activating AMPK-dependent autophagy," Brain Research Bulletin, vol. 139, pp. 105-113, 2018.

[15] J. B. Bederson, L. H. Pitts, M. Tsuji, M. C. Nishimura, R. L. Davis, and H. Bartkowski, "Rat middle cerebral artery occlusion: evaluation of the model and development of a neurologic examination," Stroke, vol. 17, no. 3, pp. 472476, 1986

[16] R. Liu, J. Diao, S. He et al., "XQ-1H protects against ischemic stroke by regulating microglia polarization through PPAR $\gamma$ pathway in mice," International Immunopharmacology, vol. 57, pp. 72-81, 2018.

[17] X. Li, L. Huang, G. Liu et al., "Ginkgo diterpene lactones inhibit cerebral ischemia/reperfusion induced inflammatory response in astrocytes via TLR4/NF- $\kappa \mathrm{B}$ pathway in rats," Journal of Ethnopharmacology, vol. 249, article 112365, 2020.

[18] H. X. Chu, H. A. Kim, S. Lee et al., "Immune cell infiltration in malignant middle cerebral artery infarction: comparison with transient cerebral ischemia," Journal of Cerebral Blood Flow and Metabolism, vol. 34, no. 3, pp. 450-459, 2014.

[19] R. M. Ransohoff, "A polarizing question: do M1 and M2 microglia exist?," Nature Neuroscience, vol. 19, no. 8, pp. 987-991, 2016.

[20] V. E. Miron, A. Boyd, J. W. Zhao et al., "M2 microglia and macrophages drive oligodendrocyte differentiation during
CNS remyelination," Nature Neuroscience, vol. 16, no. 9, pp. 1211-1218, 2013.

[21] C. Ren, Z. Yan, D. Wei, X. Gao, X. Chen, and H. Zhao, "Limb remote ischemic postconditioning protects against focal ischemia in rats," Brain Research, vol. 1288, pp. 88-94, 2009.

[22] W. Xu, W. Jin, X. Zhang, J. Chen, and C. Ren, "Remote limb preconditioning generates a neuroprotective effect by modulating the extrinsic apoptotic pathway and TRAIL-receptors expression," Cellular and Molecular Neurobiology, vol. 37, no. 1, pp. 169-182, 2017.

[23] M. Xia, Q. Ding, Z. Zhang, and Q. Feng, "Remote limb ischemic preconditioning protects rats against cerebral ischemia via HIF-1 $\alpha$ /AMPK/HSP70 pathway," Cellular and Molecular Neurobiology, vol. 37, no. 6, pp. 1105-1114, 2017.

[24] J. P. Michaud, P. M. Pimentel-Coelho, Y. Tremblay, and S. Rivest, "The impact of Ly6Clow monocytes after cerebral hypoxia-ischemia in adult mice," Journal of Cerebral Blood Flow \& Metabolism, vol. 34, no. 7, pp. e1-e9, 2014.

[25] D. J. Loane and A. Kumar, "Microglia in the TBI brain: the good, the bad, and the dysregulated," Experimental Neurology, vol. 275, Part 3, pp. 316-327, 2016.

[26] J. Y. Kim, N. Kim, and M. A. Yenari, "Mechanisms and potential therapeutic applications of microglial activation after brain injury," CNS Neuroscience \& Therapeutics, vol. 21, no. 4, pp. 309-319, 2015.

[27] S. Girard, D. Brough, G. Lopez-Castejon, J. Giles, N. J. Rothwell, and S. M. Allan, "Microglia and macrophages differentially modulate cell death after brain injury caused by oxygen-glucose deprivation in organotypic brain slices," Glia, vol. 61, no. 5, pp. 813-824, 2013.

[28] S. C. He, R. Liu, B. B. Li et al., "Propagermanium, a CCR2 inhibitor, attenuates cerebral ischemia/reperfusion injury through inhibiting inflammatory response induced by microglia," Neurochemistry International, vol. 125, pp. 99110, 2019.

[29] Y. Wang, Y. Huang, Y. Xu et al., "Correction to: A dual AMPK/Nrf2 activator reduces brain inflammation after stroke by enhancing microglia M2 polarization," Antioxidants \& Redox Signaling, vol. 32, no. 3, pp. 213-214, 2020.

[30] Z. H. Qin, L. Y. Tao, and X. Chen, "Dual roles of NF- $\kappa$ B in cell survival and implications of NF- $\kappa$ B inhibitors in neuroprotective therapy," Acta Pharmacologica Sinica, vol. 28, no. 12, pp. 1859-1872, 2007.

[31] T. Lawrence, "The nuclear factor NF-kappaB pathway in inflammation," Cold Spring Harbor Perspectives in Biology, vol. 1, no. 6, article a001651, 2009.

[32] A. R. Carta, A. Pisanu, and E. Carboni, "Do PPAR-gamma agonists have a future in Parkinson's disease therapy?," Parkinson's disease, vol. 2011, article 689181, pp. 1-14, 2011.

[33] A. Pisanu, D. Lecca, G. Mulas et al., "Dynamic changes in proand anti-inflammatory cytokines in microglia after PPAR- $\gamma$ agonist neuroprotective treatment in the MPTPp mouse model of progressive Parkinson's disease," Neurobiology of Disease, vol. 71, pp. 280-291, 2014.

[34] M. I. Cuartero, I. Ballesteros, A. Moraga et al., "N2 neutrophils, novel players in brain inflammation after stroke," Stroke, vol. 44, no. 12, pp. 3498-3508, 2013.

[35] M. A. Bouhlel, B. Derudas, E. Rigamonti et al., "PPAR $\gamma$ activation primes human monocytes into alternative M2 macrophages with anti-inflammatory properties," Cell Metabolism, vol. 6, no. 2, pp. 137-143, 2007. 
[36] A. M. Fagenson, K. Xu, F. Saaoud et al., "Liver ischemia reperfusion injury, enhanced by trained immunity, is attenuated in caspase 1/caspase 11 double gene knockout mice," Pathogens, vol. 9, no. 11, 2020.

[37] Y. Shao, J. Saredy, W. Y. Yang et al., "Vascular endothelial cells and innate immunity," Arteriosclerosis, Thrombosis, and Vascular Biology, vol. 40, no. 6, pp. e138-e152, 2020. 